\title{
CP DECOMPOSITION AND ITS APPLICATION IN NOISE REDUCTION AND MULTIPLE SOURCES IDENTIFICATION
}

\author{
Xuefeng LIU, Yuping FENG, Ximei LIU, Biao HUANG \\ College of Automation \& Electronic Engineering, \\ Qingdao University of Science \& Technology, China
}

\begin{abstract}
The CANDECOMP/PARAFAC (Canonical Decomposition / Parallel Factor Analysis, ab. CP) decomposition of a higherorder tensor is a powerful multilinear algebra, thus denoising observed data and identification of multiple sources can both be accomplished by the CP decomposition. In this paper, images are used to illustrate the denoising effect of $\mathrm{CP}$ decomposition. To identify sources, the number of senors affects the accuracy and convergence of algorithm greatly, especially, if the angle difference is smaller the problem would be worse. So, we concern to improve the accuracy of the identification of close sources with $\mathrm{CP}$ decomposition. By searching for the optimal number of sensors and denoising the original data by $\mathrm{CP}$ beforehand, the fewer number of sensors could be used and the accuracy is improved. Simulation results show that our technique outperforms good performances in both denoising data and identification of close sources.
\end{abstract}

Keywords: CP Decomposition, Noise Reduction, Multiple Sources Identification, Identification, Optimal Number of Sensors.

\section{INTRODUCTION}

In the last 10 years, interest in tensor decompositions has expanded to many fields, such as signal processing [1], numerical linear algebra [3], computer vision [4], numerical analysis [5], data mining [6], graphic analysis [7], neuroscience, communication and so on. Moreover, the adaptive algorithms to track $\mathrm{CP}$ decomposition is also researched.

The distinguishing characteristic of $\mathrm{CP}$ is its uniqueness properties, that is to say that low-rank CP decomposition can be unique for rank higher than one, and this is a key strength of CP. Our approach herein is to verify that CP model can be used to denoise color images and the denoised results show the promising prospects of this algorithm in this field.

Estimation of Direction Of Arrival (DOA) is the key problem in emitter localization and is central to the identification of multiple sources in a sensor array system. Like MUSIC (Multiple Signal Classification) [13] and ESPRIT (Estimation of Signal Parameters via Rotational Invariance Technique), CP analysis has been successfully introduced to DOA estimation in sensor array processing [8] and applied in detection and localization of multiple sources in a MIMO radar system [9]. But for close sources the accuracy and the convergence of algorithm would be affect greatly by the number of senors, especially for the small angle difference such as $1^{\circ}$. For improving the accuracy of the identification of close sources with $\mathrm{CP}$ algorithm and reducing the numbers of sensors, we denoise the observed data by $\mathrm{CP}$ model in advance so that the SNR can be ameliorated, then the fewer number of sensor could be used to get the same accuracy or the better accuracy will be gained with the same number of sensors. The simulation results show our technique does good job in this field.

The rest of this paper is structured as follow. Some multilinear algebra prerequisites are introduced in Section 2. Section 3 introduces the $\mathrm{CP}$ decomposition and describes how this model denoises data, to visibly illustrate the denoising effect some color images are applied. Whereas Section 5 illustrates the numerical results of the identification with optimal number of sensors and the improvement after CP denoising. Conclusions are drawn in Section 6.

Notation: An $N$-way tensor of size $I_{1} \times I_{2} \times \ldots I_{N}$ is denoted by a calligraphic letter $\mathscr{X}$ and its elements are denoted by $x_{i_{1}, \ldots, i_{N}}, i_{1}=1, \ldots, I_{1}, i_{2}=1, \ldots, I_{2}$, and $i_{N}=1, \ldots, I_{N}$. A italic capital letter $X$ denotes a matrix and a italic lower-case letter $\boldsymbol{x}$ a vector. The transpose is denoted by $X^{\mathrm{T}} .\|X\|$ is the Frobenius norm of $X$. The Kronecker product is denoted by $\otimes$. The Khatri-Rao product (or column-wise Kronecker product) is denoted by $\odot$.

\section{MULTILINEAR ALGEBRA PREREQUISITES}

\subsection{Rank-one tensors}

$N$-mode tensor $\mathscr{X} \in \mathbb{R}^{I_{1} \times I_{2} \times \ldots \times I_{N}}$ being rank 1 means that it can be written as the outer product of $N$ vectors, i.e.

$$
\mathscr{X}=\boldsymbol{a}^{(1)} \circ \boldsymbol{a}^{(2)} \circ \ldots \circ \boldsymbol{a}^{(N)}
$$

So, each element of $\mathscr{X}$ is the product of the corresponding vector elements:

$$
x_{i_{1}, i_{2}, \ldots, i_{N}}=a_{i_{1}}^{(1)} a_{i_{2}}^{(2)} \ldots a_{i_{N}}^{(N)} \text { for all } 1 \leq i_{n} \leq I_{n}
$$

\subsection{Tensor multiplication}

We consider only the tensor $n$-mode product here, i.e., multiplying a tensor $\mathscr{X} \in \mathbb{R}^{I_{1} \times I_{2} \times \ldots \times I_{N}}$ by a matrix (or a vector) $U \in \mathbb{R}^{J \times I_{N}}$ in mode $n$. It is denoted by $\mathscr{X} \times{ }_{n} U$ and is of size $I_{1} \times \ldots \times I_{n-1} \times J \times I_{n+1} \times \ldots \times I_{N}$. Elementwise, we have

$$
\left(\mathscr{X} \times{ }_{n} U\right)_{i_{1} \ldots i_{n-1} j i_{n+1} \ldots i_{N}}=\sum_{i_{n}=1}^{I_{n}} x_{i_{1} i_{2} \ldots i_{N}} u_{j i_{n}}
$$

In terms of unfolded tensors the idea can also be expressed as:

$$
y=\mathscr{X} \times_{n} U \Longleftrightarrow Y_{(n)}=U X_{(n)}
$$




\subsection{Matrix Kronecker and Khatri-Rao products}

The Kronecker product of matrices $A \in \mathbb{R}^{I \times J}$ and $B \in \mathbb{R}^{M \times N}$ is a matrix of size $(I M) \times(J N)$ and defined by

$$
\begin{aligned}
& A \otimes B=\left[\begin{array}{cccc}
a_{11} B & a_{12} B & \cdots & a_{1 J} B \\
a_{21} B & a_{22} B & \cdots & a_{2 J} B \\
\vdots & \vdots & \ddots & \vdots \\
a_{I 1} B & a_{I 2} B & \cdots & a_{I J} B
\end{array}\right]
\end{aligned}
$$

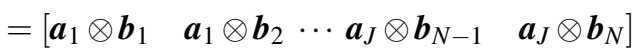

A column-wise Kronecker product of two matrices is called the Khatri-Rao product [10]. The Khatri-Rao product of matrices $A \in \mathbb{R}^{I \times M}$ and $B \in \mathbb{R}^{J \times M}$ is a matrix of size $(I J) \times M$ and defined by

$$
A \odot B=\left[\begin{array}{llll}
\boldsymbol{a}_{1} \otimes \boldsymbol{b}_{1} & \boldsymbol{a}_{2} \otimes \boldsymbol{b}_{2} & \cdots & \boldsymbol{a}_{M} \otimes \boldsymbol{b}_{M}
\end{array}\right]
$$

\section{CP DECOMPOSITION}

$\mathrm{CP}$ decomposition relies on the definition of rank of the tensor : each rank $R$ tensor $\mathscr{X} \in \mathbb{R}^{I_{1} \times I_{2} \times \ldots \times I_{N}}$ can be a sum of rank-1 tensors

$$
\mathscr{X}=\sum_{r=1}^{R} \mathscr{X}_{r}=\sum_{r=1}^{R} \boldsymbol{u}_{r}^{(1)} \circ \ldots \circ \boldsymbol{u}_{r}^{(N)}
$$

where $\forall r=1, \ldots, R, \mathscr{X}_{r} \in \mathbb{R}^{I_{1} \times I_{2} \times \ldots \times I_{N}}$ is rank 1 and $\forall n=$ $1, \ldots, N, u_{r}^{(n)} \in \mathbb{R}^{I_{n}}$ is a base vector of the $n$-modal space of $\mathscr{X}$.

The rank $K$ approximation of a rank $R$ is :

$$
\hat{\mathscr{X}}=\sum_{r=1}^{K} \mathscr{X}_{r}=\sum_{r=1}^{K} \boldsymbol{u}_{r}^{(1)} \circ \ldots \circ \boldsymbol{u}_{r}^{(N)}
$$

while minimizing the square error $\|\mathscr{X}-\hat{\mathscr{X}}\|^{2}$. Using Khatri-Rao product, (8) can be written $\forall n=1, \ldots, N$ as :

$$
\hat{\mathscr{X}}=U^{(n)}\left(U^{(1)} \odot \ldots \odot U^{(n-1)} \odot U^{(n+1)} \odot \ldots \odot U^{(N)}\right)^{T}
$$

where $U^{(n)}=\left[\boldsymbol{u}_{1}^{(1)}, \ldots, \boldsymbol{u}_{K}^{(N)}\right]$. Thus, the minimization of $\|\mathscr{X}-\hat{X}\|^{2}$ comes to find $U^{(n)}$ which makes the square error the minimum:

$$
\begin{aligned}
e\left(U^{(1)} \ldots U^{(N)}\right)= & \| \hat{\mathscr{X}}-U^{(n)}\left(U^{(1)} \odot \ldots \odot U^{(n-1)}\right. \\
& \left.\odot U^{(n+1)} \odot \ldots \odot U^{(N)}\right)^{T} \|^{2}
\end{aligned}
$$

An ALS process $[11,12]$ can solve such a problem.

\section{DENOISING BASED ON CP DECOMPOSITION}

\subsection{Denoising process}

Given that observed data $\mathscr{X} \in \mathbb{R}^{I_{1} \times I_{2} \times I_{3}}$ consist of a signal tensor $S \in \mathbb{R}^{I_{1} \times I_{2} \times I_{3}}$ and an additive noise tensor $N \in$ $\mathbb{R}^{I_{1} \times I_{2} \times I_{3}}$ :

$$
\mathscr{X}=S+N
$$

If the rank of $S$ is $K$ and equals to the rank of $\mathscr{X}$, the $K$-approximation of $\mathscr{X}$ will be (8). According to (10) $\forall n=$ $1, \ldots, N$, we can write:

$$
U^{(n)}=\mathscr{X}_{n} Z^{(n)}\left(Z^{(n)^{T}} Z^{(n)}\right)^{\sharp}
$$

where $Z^{(n)}$ is the Khatri-Rao product of all matrixes $U$ without $U^{(n)}$ described in CP ALS algorithm [11]. Then, (12) can be introduced in the flattened expression:

$$
\hat{S}_{n}=\hat{\mathscr{X}}_{n} P^{(n)^{T}}
$$

where $P^{(n)}=Z^{(n)^{T}}\left(Z^{(n)} Z^{(n)^{T}}\right)^{\sharp} Z^{(n)}$. Thus, a CP decomposition can be denoted by $\left\{P^{(n)}\right\}_{n=1, \ldots, N}$, which is a set of linked projectors. Here $P^{(n)}$ (of rank $K$ ) is applied in the transposed $n$-mode flattening matrix of the tensor. So the previous $K$ approximation of the noisy tensor $\mathscr{X}$ can be written as:

$$
\hat{S}_{n}=\hat{\mathscr{X}}_{n} P^{(n)^{T}}=S_{n} P^{(n)^{T}}+N_{n} P^{(n)^{T}}
$$

Those $P^{(n)}$ perform function of rank reduction on each signal. If it has full rank, the noise signal will be generally decreased when $K<\max \left(I_{1}, I_{2}, I_{3}\right)$. As a result, a low rank $\mathrm{CP}$ approximation of a noisy tensor reduces noise and estimates the signal.

\subsection{Denoising results}

As visible examples, we apply the $\mathrm{CP}$ algorithm into the denoising of two color images. In the first case, four kinds of different noises, i.e. Gaussian, Poisson, Salt \& Pepper and Speckle noises, are add to the original image respectively. Next, to evaluate quantitatively the results obtained by $\mathrm{CP}$ model, the signal to noise ratio (SNR) is defined as

$$
\mathrm{SNR}=10 \log _{10} \frac{\|S\|^{2}}{\|N\|^{2}}[\mathrm{~dB}]
$$

And six values of $\operatorname{SNR}(0,5,10,15,20$ and $25 \mathrm{~dB})$ are fixed to each noisy image. Then, to evaluate the quality of the estimated signal tensor, the normalized quadratic error (NQE) criterion is calculated by

$$
\operatorname{NQE}(\hat{\mathscr{X}})=\|\hat{\mathscr{X}}-\mathscr{X}\|^{2} /\|\mathscr{X}\|^{2}
$$

In the end, the optimal rank is selected at each SNR according to the minimum NQE, as shown in Fig.1.

It is clear in Fig. 1 that the optimal rank of CP denoising in Mondrian noisy image is much lower than Lena two image at the same SNR, because there are 2 distinct orthogonal directions in the original Mondrian image and the $\mathrm{CP}$ denoising is to decompose the high-order tensor to rank-1 ones, they are consistent in the sense of low-rank decomposition.

Fig. 2 shows the denoising result of images with Gaussian noise at SNR of $10 \mathrm{~dB}$ and the optimal rank and NQE of CP denoising are illustrated in Table 1.

Table 1: Optimal rank and NQE

\begin{tabular}{|c|c|c|}
\hline Image & Optimal rank & NQE $\left(\times 10^{-2}\right)$ \\
\hline Lena & 40 & 6.28 \\
\hline Mondrian & 11 & 5.65 \\
\hline
\end{tabular}

In the next section we give identification results concerning the localization of the narrow-band sources. 


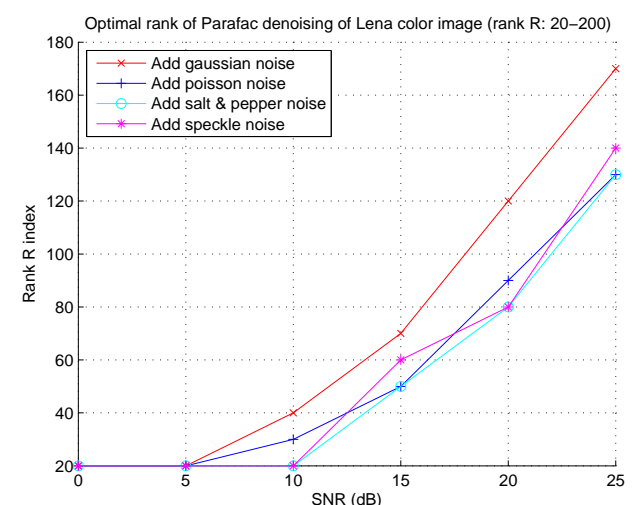

(a)

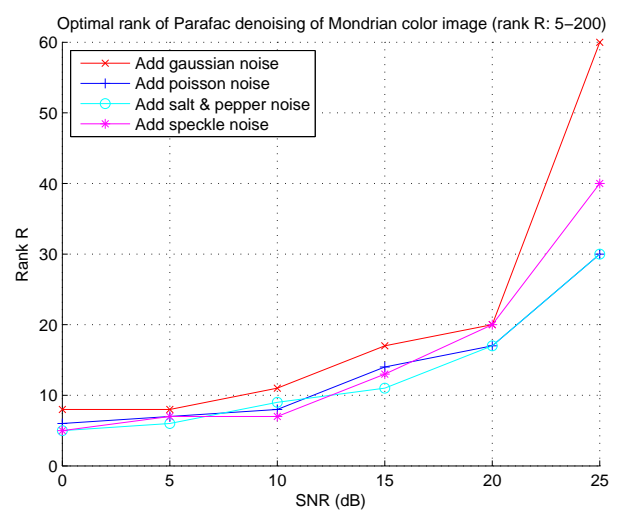

(b)

Figure 1: Optimal rank of CP denoising results. (a) Lena color image, (b) Mondrian color image.

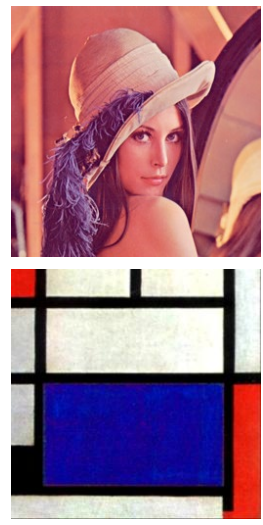

(a)

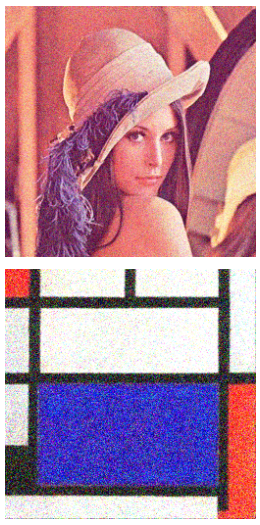

(b)

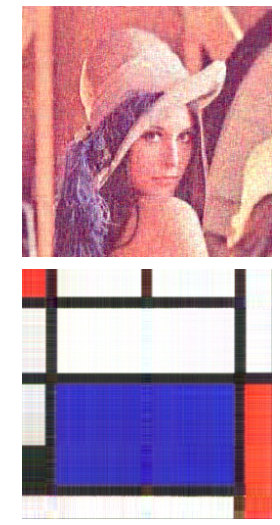

(c)
Figure 2: CP denoising results. (a) are the original images; (b) are the noisy images with the Gaussian noise of $\mathrm{SNR}=10 \mathrm{~dB}$; (c) are the denoising results with $\mathrm{CP}$ model. The rows show the results of Lena and Mondrian color images respectively.

\section{IMPACT OF THE OPTIMAL NUMBER OF SENSORS IN MULTIPLE SOURCES IDENTIFICATION}

\subsection{Optimal number of sensors}

Suppose that the number of sources is known and satisfies the uniqueness of $\mathrm{CP}$ decomposition of $\mathscr{X}$, different sources could be identified by estimating their DOA [8]. Given the received signal is:

$$
\hat{\mathscr{X}}=(A \odot S) \Phi^{T}+Z
$$

where $\hat{\mathscr{X}}$ is the receiving data of sensor array, $A$ is subarray response, the diagonal of $\Phi$ depends on source parameters, $S$ is the source signal matrix and $Z$ is the noise matrix. According to the CP model, we can get matrix $A$ and $\Phi$ and further DOA.

If the angle difference is small, for instance $1^{\circ}$, the results will not be so stable. Increasing the number of sensors is a solution to the problem. Another way to solve it is to select the optimal number of sensors when doing CP decomposition so than the localization accuracy of close sources could be further improved. That is to say, with the optimal number of sensors, the close sources (the smallest angle difference is $1^{\circ}$ ) could be well separated and localized at the best accuracy. At different SNRs, the optimal numbers of sensors with angle difference from $1^{\circ}$ to $5^{\circ}$ are showed in Fig. 3, here $A$ and $\Phi$ (which are described in the next subsection) are extracted from the response array [8].

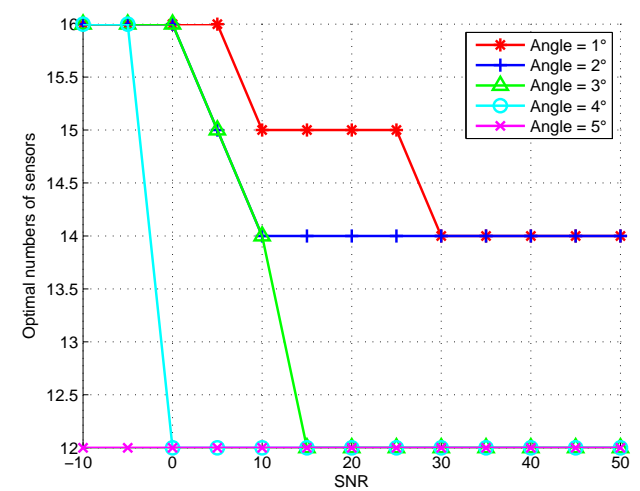

(a)

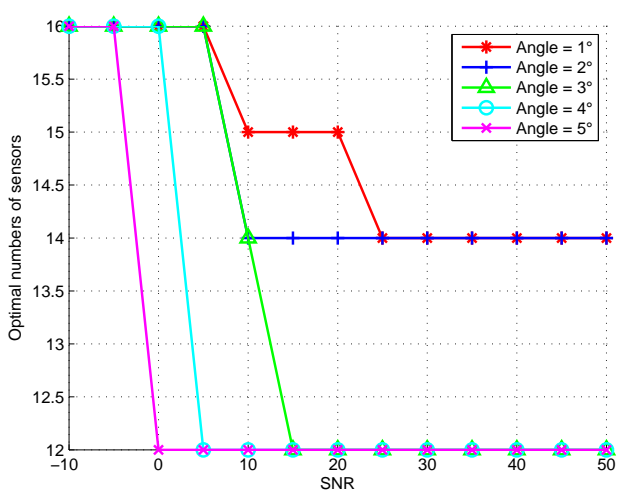

(b)

Figure 3: Optimal numbers of sensors to identify close sources by CP. (a) optimal numbers of sensors from $A$ of CP decompostiion, (b) optimal numbers of sensors from $\Phi$ of CP decompostiion.

\subsection{Impact of the optimal number of sensors}

To further improve the accuracy or decrease the optimal number of sensors, we can denoise the observed data with CP model beforehand to ameliorate SNR. 
Table 2: Identification results of angle difference being $1^{\circ}$

\begin{tabular}{|c|c|c|c|c|c|c|}
\hline \multirow[b]{2}{*}{ N.S. } & \multicolumn{2}{|c|}{ From $A$} & \multicolumn{2}{|c|}{ From $\Phi$} & \multirow[b]{2}{*}{$\frac{\left\|\mathscr{X}_{d}\right\|^{2}}{\|\mathscr{X}\|^{2}}$} & \multirow[b]{2}{*}{$\begin{array}{l}\mathrm{SNR}(\mathrm{dB}) \\
\text { after de- } \\
\text { noising }\end{array}$} \\
\hline & $\begin{array}{l}\text { Estimated } \\
\text { angles }\end{array}$ & $\begin{array}{l}\text { Error }= \\
\left|\theta_{A}-\theta\right|\end{array}$ & $\begin{array}{l}\text { Estimated } \\
\text { angles }\end{array}$ & $\begin{array}{l}\text { Error }= \\
\left|\theta_{\Phi}-\theta\right|\end{array}$ & & \\
\hline \multirow{2}{*}{10} & 45.44 & 0.44 & 45.39 & 0.39 & \multirow{2}{*}{7.51} & \multirow{2}{*}{7.25} \\
\hline & 46.46 & 0.46 & 46.43 & 0.43 & & \\
\hline \multirow{2}{*}{15} & 45.30 & 0.30 & 45.29 & 0.29 & \multirow{2}{*}{9.00} & \multirow{2}{*}{8.28} \\
\hline & 46.51 & 0.51 & 46.53 & 0.53 & & \\
\hline \multirow{2}{*}{16} & 45.05 & 0.05 & 45.04 & 0.04 & \multirow{2}{*}{0.91} & \multirow{2}{*}{19.25} \\
\hline & 46.10 & 0.10 & 46.10 & 0.10 & & \\
\hline \multirow{2}{*}{17} & 45.03 & 0.03 & 45.05 & 0.05 & \multirow{2}{*}{5.49} & \multirow{2}{*}{15.46} \\
\hline & 45.69 & 0.31 & 45.68 & 0.32 & & \\
\hline \multirow{2}{*}{20} & 45.19 & 0.19 & 45.19 & 0.19 & \multirow{2}{*}{2.33} & \multirow{2}{*}{13.11} \\
\hline & 46.39 & 0.39 & 46.36 & 0.36 & & \\
\hline
\end{tabular}

In simulation, two sources of $45^{\circ}$ and $46^{\circ}$ (denoted by $\theta$ ) are identified at $\mathrm{SNR}=5 \mathrm{~dB}$ and the results are listed in table 2 (here N.S. means number of sensors, $\mathscr{X}$ is the tensor of observed data and $\mathscr{X}_{d}$ denotes the tensor after denoising). From Table 2 we can know that the estimated angles gained from 16 sensors are more accurate than those gained from more or less than 16 sensors. That is to say, the errors between original and estimated angles verify that the identification could be more accurate with the optimal number of sensors. And in Table 2, it is distinct that the SNR has been improved after $\mathrm{CP}$ denoising, so we can use fewer sensors to get the same accuracy of identification or obtain better accuracy with improved SNR.

For other array processing methods such as MUSIC algorithm the two sources with $1^{\circ}$ angular difference cannot be identified and only one angle is obtained around $45^{\circ}$. More importantly, CP performs well even the noise is specially correlated while MUSIC cannot get the useful results with unknown noise.

\section{CONCLUSION}

$\mathrm{CP}$ analysis has been well applied in DOA estimation in sensor array processing and in detection and localization of multiple sources in a MIMO radar system. But when angle difference is small the number of senors affected the accuracy of the results and the convergence of $\mathrm{CP}$ greatly. To improve the accuracy of the identification of close sources, we search for the optimal number of sensors with fixed SNR. And we further decrease the numbers of sensors and improve the accuracy by denoising the original data by $\mathrm{CP}$ model so that to improve the SNR. In this paper, to demonstrate the denoising effect of $\mathrm{CP}$ we take two color images as examples. The detection of multiple sources in a sensor array system shows the exact localization of $\mathrm{CP}$ and two close sources of $1^{\circ}$ angle difference are well identified after CP denoising. Our approach herein is to prove that the accuracy of identification could be gained by selecting optimal number of sensors and could be further improved by $\mathrm{CP}$ denoising in advance. Through the application in images we demonstrate the excellent denoising capability of CP model and the results of localization and identification also show it is preferable in identification in sensor array processing.

\section{Acknowledgement.}

The work was supported by the National Natural Science Foundation of China (Grant No. 61401244), Shandong Provincial Natural Science Foundation, China (Grant No. ZR2014FM013) and the Taishan Scholar Program of Shandong.

\section{REFERENCES}

[1] D. Muti and S. Bourennane, "Multidimensional filtering based on a tensor approach," Signal Processing, vol. 85, pp. 2338-2353, 2005.

[2] L. De Lathauwer, J. Castaing and J.-F. Cardoso, "Fourth-order cumulant based blind identification of underdetermined mixtures," IEEE Transactions on Signal Processing, vol. 55, pp. 2965-2973, 2007.

[3] A. N. Langville and W. J. Stewart, "A kronecker product approximate precondition for sans," Numerical Linear Algebra with Applications, vol. 11, pp. 723-752, 2004.

[4] T. Hazan, S. Polak and A. Shashua, "Sparse image coding using a $3 \mathrm{~d}$ non-negative tensor factorization," ICCV 2005: Proceedings of the 10th IEEE International Conference on Computer Vision, vol. 1. IEEE Computer Society, 2005, pp. 50-57.

[5] B. N. Khoromskij and V. Khoromskaia, "Low rank tucker-type tensor approximation to classical potentials," Central European Journal of Mathematics, vol. 5, pp. 523-550, 2007.

[6] B. W. Bader, M. W. Berry and M. Browne, Discussion tracking in enron email using PARAFAC, 2nd ed. Springer, 2007, ch. Survey of Text Mining: Clustering, Classification, and Retrieval, pp. 147-162.

[7] B. W. Bader, R. A. Harshman and T. G. Kolda, "Temporal analysis of semantic graphs using asalsan," ICDM 2007: Proceedings of the 7th IEEE International Conference on Data Mining, Oct. 2007, pp. 33-42.

[8] Nicholas D. Sidiropoulos, Rasmus Bro and G. B. Giannakis, "Parallel factor analysis in sensor array processing," IEEE Transactions on Signal Processing, vol. 48, no. 8, Aug. 2000.

[9] D. Nion and N. D. Sidiropoulos, "A parafac-based technique for detection and localization of multiple targets in a mimo radar system," 2009 IEEE International Conference on Acoustics, Speech and Signal Processing, April 2009, pp. 2077-2080.

[10] A. Smilde, R. Bro and P. Geladi, Multi-Way Analysis: Applications in the Chemical Sciences. Wiley, West Sussex, England, 2004.

[11] Bro R., "Multiway analysis in the food industry," Ph.D. dissertation, Royal Vetenary and Agricultural University, Denmark, 1998.

[12] X. Liu, S. Bourennane and C. Fossati, "Denoising of Hyperspectral Images Using the PARAFAC Model and Statistical Performance Analysis," IEEE Transactions on Geoscience and Remote Sensing, vol. 50, pp. 37173724, 2012.

[13] Cadzow J. A., "A high resolution direction-of-arrival algorithm for narrow-band coherent and incoherent 
sources", IEEE Transactions on Acoustics, Speech, and

Signal Processing, vol.36, no.7, pp. 965-979, 1988. 